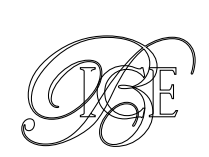

\title{
SUPRESIÓN DE BARRERAS A LA INDUSTRIA FARMACÉUTICA DE LA UE: OPORTUNIDADES Y RETOS
}

Recientemente se ha aprobado el Reglamento (UE) 2019/933 del Parlamento Europeo y del

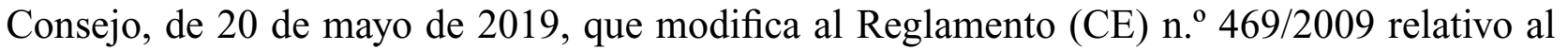
certificado complementario de protección para los medicamentos. Este artículo explica en qué ha consistido este cambio legislativo en materia de propiedad industrial en el sector farmacéutico, los motivos que han impulsado la modificación y cuáles podrían ser las consecuencias en términos de tejido industrial y de competencia en este sector en la UE.

Palabras clave: propiedad industrial, patentes, industria farmacéutica, competitividad, medicamentos, genéricos, biosimilares.

Clasificación JEL: L65, O34.

\section{Introducción}

La evolución del mercado farmacéutico a nivel mundial ha hecho que la Unión Europea (UE) se enfrente a una creciente competencia desde el exterior, especialmente en el sector en plena expansión de los medicamentos biosimilares $^{1}$ (Comisión Europea, 2018; Navarro, 2007).

1 Un medicamento biosimilar se desarrolla con la intención de ser muy parecido a su medicamento de referencia en términos de calidad, seguridad y eficacia. El principio activo de un medicamento biosimilar y el de su medicamento de referencia son, en esencia, la misma sustancia biológica, aunque pueden existir entre ellos ligeras diferencias derivadas de su naturaleza compleja y de sus métodos de producción. Medicamento

\footnotetext{
* Funcionaria del Cuerpo de Farmacéuticos Titulares. Consejera Técnica en la Dirección General de Política Económica, Ministerio de Economía y Empresa.

Versión de septiembre de 2019.

DOI: https:/doi.org/10.32796/bice.2019.3115.6907

Las informaciones y opiniones vertidas en este artículo se realizan a título personal y no vinculan en ningún caso al Ministerio de Economía y Empresa.
}

Es bien conocido que la primera empresa en comercializar un medicamento genérico o biosimilar adquiere una cuota muy elevada del mercado $^{2}$. En consecuencia, se ha modificado la normativa europea con el fin de que las empresas ubicadas en la UE sean más competitivas, inviertan en plantas de producción en nuestro territorio y no deslocalicen sus fábricas.

El sector farmacéutico es un sector industrial de alto riesgo ${ }^{3}$ y con una actividad de elevado valor estratégico dada su contribución a la salud. Por ello, en este sector cobra gran relevancia la protección de la innovación.

de referencia es el medicamento original o innovador que obtuvo la autorización de comercialización con base en un dosier de registro completo y que está autorizado desde hace ocho años como mínimo por un Estado miembro de la UE o en la UE por procedimiento centralizado.

2 Entre otros, lo expone la OCDE en sus estudios sectoriales como los de mesas redondas sobre políticas de medicamentos genéricos.

3 Por los elevados costes de $1+\mathrm{D}$, el alto riesgo de fracaso de investigación y ser un sector ampliamente regulado, con elevadas exigencias técnicas, necesidad de autorización previa a la comercialización, etcétera. 
Las empresas en el mercado farmacéutico protegen la innovación mediante patentes. La patente es un título jurídico que puede otorgarse por una invención de carácter técnico siempre que sea nueva, entrañe una actividad inventiva y pueda tener una aplicación industrial. Una patente da derecho al propietario a impedir que otros fabriquen, utilicen o vendan la invención sin permiso. Así, las patentes animan a las empresas a hacer la inversión necesaria en innovación y funcionan como incentivo para que las personas inversoras y las empresas dediquen recursos a la investigación y al desarrollo. En Europa, las invenciones técnicas pueden protegerse mediante patentes nacionales, concedidas por las autoridades nacionales competentes, o mediante patentes europeas, concedidas de manera centralizada por la Oficina Europea de Patentes (OEP).

Los medicamentos innovadores en España se protegen mediante patente nacional, y ésta se regula a través de la Ley 24/2015, de 24 de julio, de Patentes.

En adición a la patente existe un Certificado Complementario de Protección (CCP o SPC - siglas en inglés-), para el ámbito del medicamento ${ }^{4}$, en la UE. Este certificado se creó en 1992, la solicitud y el correspondiente procedimiento de concesión del CCP son de carácter nacional ${ }^{5}$ y se regula en la actualidad por el Reglamento (CE) n. ${ }^{-469 / 2009}$ del Parlamento Europeo y del Consejo, de 6 de mayo de 2009, relativo al certificado complementario de protección para los medicamentos, norma que ha sido recientemente modificada.

El objetivo del CCP es el de compensar al titular el tiempo perdido de la vida de la patente,

\footnotetext{
4 Título industrial exclusivamente para productos farmacéuticos y fitosanitarios.

5 Por ello su solicitud ha de efectuarse en cada Estado miembro para el que se desee tener la protección.
}

al no poder poner el producto en el mercado hasta no haber obtenido la autorización de comercialización por parte de las autoridades sanitarias.

Los derechos de un CCP solamente se extienden al producto amparado por la autorización de comercialización como medicamento. La protección del CCP no se extiende más que a las utilizaciones farmacéuticas autorizadas del producto y no protege otros eventuales usos no farmacéuticos.

EI CCP es un título que tiene efecto desde la fecha de expiración de la patente de base, y se concede por el tiempo que transcurre entre la fecha de solicitud de la patente y la fecha de la primera autorización de comercialización en la UE, restándole 5 años, que es el tiempo usual de tramitación de la solicitud de patente, y con la limitación de que no puede tener una duración superior a 5 años. Sólo será de duración mayor, 6 meses adicionales, si se concede una prórroga pediátrica ${ }^{6}$. En la práctica, supone tener una exclusividad en el mercado de unos 10 a 15 años.

En paralelo a la patente, existe la protección de datos que aplican las autoridades sanitarias. Dicha exclusividad se regula en la Directiva 2001/83/CE, código comunitario sobre medicamentos para uso humano ${ }^{7}$, y a nivel nacional en el texto refundido de la Ley de Garantías y Uso Racional de los Medicamentos y Productos Sanitarios, aprobado por el Real Decreto Legislativo 1/2015, de 24 de julio. Para que las agencias de medicamentos acepten como válida $\triangleright$

\footnotetext{
6 En el Reglamento (CE) n. $-1901 / 2006$ del Parlamento Europeo y del Consejo, de 12 de diciembre de 2006, se establece que las empresas deberán elaborar planes de investigación pediátrica con datos sobre el uso pediátrico de un medicamento cuando soliciten la autorización para comercializar sus productos y que a cambio, como incentivo, la empresa reciba una prórroga de seis meses de su certificado complementario de protección.

7 Directiva 2001/83/CE del Parlamento Europeo y del Consejo, de 6 de noviembre de 2001, por la que se establece un código comunitario sobre medicamentos para uso humano (artículo 10).
} 
una solicitud de autorización de un medicamento genérico o biosimilar, que se basa en la evidencia de eficacia y seguridad del correspondiente medicamento innovador o de referencia, éste debe llevar ya un cierto número de años en el mercado europeo. De esta manera, se protegen los derechos de exclusiva del innovador y se comercializan genéricos de medicamentos con una amplia experiencia de uso y un conocimiento amplio de sus características y de su perfil beneficio/riesgo (García, 2010; Laguna, 2006).

Por tanto, es importante distinguir en la exclusividad de comercialización de un medicamento entre la patente, que es un concepto industrial que otorga el Ministerio de Industria, Oficina Española de Patentes y Marcas (OEPM), por un periodo de 20 años, y el periodo de protección de datos que le compete al Ministerio de Sanidad, Agencia Española de Medicamentos y Productos Sanitarios (AEMPS), de 10 años desde la autorización de comercialización (que inicia dentro del periodo de protección de la patente). Un medicamento genérico o biosimilar deberá cumplir ambos preceptos para su lanzamiento al mercado.

Coinciden genéricos y biosimilares en los preceptos respecto a los periodos otorgados por la patente y por la exclusividad de datos de las agencias de medicamentos, si bien son dos grupos de medicamentos con grandes diferencias.

Respecto a las barreras de entrada en el mercado farmacéutico, la más importante para la fabricación y comercialización de medicamentos la constituye la regulación existente en el sector farmacéutico. Al mismo tiempo, al mercado farmacéutico le afecta la regulación de la propiedad intelectual. Las patentes, al igual que los demás derechos de propiedad intelectual en sentido amplio, son monopolios legales y, como tales, configuran barreras de $\triangleright$

GRÁFICO 1

PRINCIPALES DIFERENCIAS ENTRE MEDICAMENTOS GENÉRICOSY BIOSIMILARES

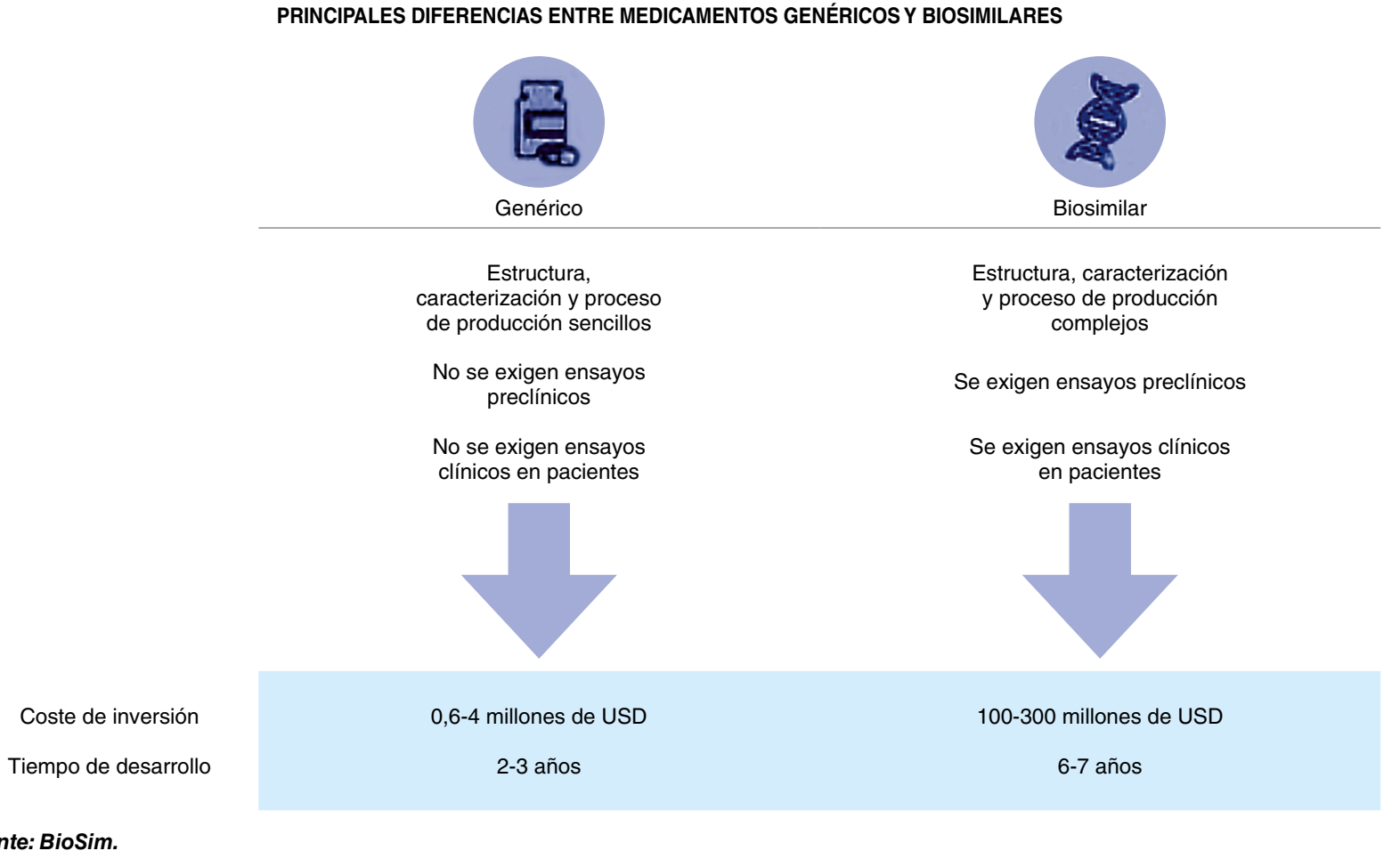


entrada a la competencia que, en casos determinados como es el mercado farmacéutico, están justificadas por sus efectos positivos.

En ocasiones, el sistema de patentes y regulatorio ha tenido efectos negativos al producirse en el mercado prácticas empresariales restrictivas de la competencia. Se han sancionado por la Comisión Europea conductas consistentes en un abuso del sistema de patentes y regulatorio y en acuerdos de patentes entre empresas (Sauri, 2015; Laguna, 2012).

Recientemente se ha aprobado el Reglamento (UE) 2019/933 del Parlamento Europeo y del Consejo, de 20 de mayo de 2019, que modifica el anteriormente citado (469/2009). Este artículo tiene como objeto explicar en qué ha consistido este cambio legislativo en el sector farmacéutico en materia de propiedad industrial, los motivos que han impulsado la modificación, que elimina barreras regulatorias para las empresas fabricantes de medicamentos genéricos y/o biosimilares, y cuáles podrían ser las consecuencias en términos de tejido industrial y de competencia en este sector en la Unión Europea.

\section{Análisis previo a la modificación legislativa}

\subsection{Estrategia para el mercado único}

El mercado único es uno de los principales logros de Europa. En el ámbito industrial, ha creado para las empresas europeas nuevas oportunidades y economías de escala que han reforzado su competitividad y ha contribuido a mejorar la integración de dichas empresas en las cadenas de valor internacionales, reforzando así su competitividad mundial. Pero tanto la UE como el mercado único han de adaptarse a un entorno cambiante. El sector farmacéutico en la UE se ha favorecido de una gran protección de la innovación, mayor que la de países de nuestro entorno. Pese a ello, en los últimos años, se ha producido una expansión del mercado farmacéutico de genéricos y biosimilares, por el gran incremento de la demanda a nivel mundial de estos medicamentos. A su vez, nos encontramos ante la expiración de un importante número de patentes de medicamentos de gran consumo en 2020 , fenómeno que se conoce como un precipicio de patentes o patent cliff.

Así, la Estrategia para el Mercado Único de 2015 anunció un reajuste selectivo de determinados aspectos de la protección, por medio de patentes y CCP, para fomentar la competitividad de industrias reguladas como la farmacéutica (Comisión Europea, 2015).

Algunos de los problemas detectados se han abordado con el nuevo reglamento. Estos son la pérdida de mercados de exportación, que son ejemplo de nuevas oportunidades empresariales para el sector, y la ausencia de una entrada oportuna en tiempo en los mercados de los Estados miembros, es decir, desde el primer día tras la expiración del CCP, para los fabricantes de genéricos y biosimilares establecidos en la UE debido a los efectos no intencionados del actual régimen del CCP de la UE.

Los otros problemas refieren a la aplicación fragmentada del régimen del CCP y de la exención de patente «Cláusula Bolar»8 en los Estados miembros, que podría solucionarse en conexión con la futura patente unitaria de la UE y con la posible creación posteriormente de un CCP unitario.

8 Dicha cláusula señala que la realización de los estudios y ensayos necesarios para el registro de un medicamento no se considerarán contrarios a los derechos de patentes ni a los CCP para medicamentos. 


\subsection{Evaluación de impacto de la Comisión Europea}

La Comisión Europea señaló que, si bien los beneficios de un CCP son considerables para su titular, el sistema del certificado, debido a los cambios significativos producidos en los mercados farmacéuticos, estaba teniendo consecuencias colaterales no deseadas para la competitividad de los fabricantes de genéricos y biosimilares establecidos en la UE. Los principales problemas detectados eran:

1. Durante el periodo de validez del certificado no se podía fabricar genéricos y biosimilares para exportarlos a países no pertenecientes a la UE en los que la protección hubiese expirado.

2. No estaban en condiciones de comercializar genéricos y biosimilares en la UE en cuanto expirase el certificado.

Tales circunstancias hacían que esos fabricantes, a diferencia de los fabricantes ubicados en terceros países en los que esta protección no existía o había expirado, tuviesen más dificultades para entrar en el mercado de la UE inmediatamente después de la expiración del $\mathrm{CCP}$, puesto que, hasta que la protección conferida por el certificado no expiraba, no se encontraban en una posición que les permitiese ampliar su capacidad de producción para fines de exportación y para fines de acceso al mercado de un Estado miembro.

La iniciativa de modificación del reglamento restablecería unas condiciones de competencia equitativas entre los fabricantes de genéricos y biosimilares establecidos en la UE y los establecidos en terceros países (donde la protección industrial es menor o no existe), lo que resultaría beneficioso para la competitividad de los primeros y de la economía europea en su conjunto, manteniendo al mismo tiempo el elevado nivel de protección de la propiedad intelectual que existe actualmente en la UE.

Sobre la magnitud exacta de los beneficios de la iniciativa existían diversas opiniones ${ }^{9}$. Se estimó, en función de una muestra de moléculas que representa el $32 \%$ de los medicamentos pertinentes, que la introducción de una dispensa para fines de exportación podría conllevar un aumento neto de las ventas de exportación de los medicamentos fabricados en la UE de entre 600 y 1.000 millones de euros anuales. Asimismo, conllevaría todos los beneficios obvios en términos de creación de empleo, estimados en 20.000-25.000 empleos directos, en función de la misma muestra limitada, y de reducción de la relocalización de plantas de producción (Comisión Europea, 2018; Consejo de la UE, 2019a).

El beneficio para las pequeñas y medianas empresas (pymes) fabricantes de genéricos y biosimilares establecidas en la UE se veía claro, dado que tienen más dificultades que las grandes empresas para crear instalaciones de fabricación fuera de la UE. Igualmente, se buscaba generar nuevas oportunidades de I+D y de fabricación para pymes y empresas emergentes en sectores sumamente lucrativos que crecen con rapidez, especialmente en lo relativo a los biosimilares, en los que existe $D$

\footnotetext{
9 La evaluación de impacto de la Comisión Europea recoge aportaciones de varios estudios sobre el CCP, que se llevaron a cabo tras la publicación de la Estrategia de Mercado Único. Entre estos:

- Study and annexes on the legal aspects of Supplementary Protection Certificates in the EU, Max Planck Institute (May 2018).

- Study of the economic impact of supplementary protection certificates, pharmaceutical incentives and rewards in Europe, Copenhagen Economics (May 2018).

- Assessing the economic impacts of changing exemption provisions during patent and SPC protection in Europe, Charles Rivers Associates (October 2017).

- 25 years of SPC protection for medicinal products in Europe: Insights and challenges, M. Mejer (May 2017).

- Economic Analysis of Supplementary Protection Certificates in Europe, M. Kyle (January 2017).
} 
una fuerte inversión en I+D. La modificación normativa sería beneficiosa para el dinamismo del sector farmacéutico de la UE en su totalidad, incluyendo la creación de empresas emergentes o de nuevas líneas de negocio.

La iniciativa tenía como objetivo, como se ha expuesto, reafirmar la posición de la UE como centro de innovación farmacéutica, especialmente en el sector de los biosimilares, a la vez que buscaba beneficiar a los pacientes y a los sistemas sanitarios, acelerando el acceso a medicamentos más asequibles tras la expiración del certificado complementario de protección.

Con fecha 28 de mayo de 2018, la Comisión Europea presentó su propuesta de reglamento que modificaba el Reglamento (CE) n. $=$ 469/2009 relativo al certificado complementario de protección para los medicamentos, que seguidamente se tramitó durante meses y finalmente se aprobó el texto definitivo el 17 de abril de 2019 (Parlamento Europeo, 2019; Comisión Europea, 2019; Consejo de la UE, 2019a, b y c).

\section{Novedades que aporta el Reglamento (UE) 2019/933}

El Reglamento (UE) 2019/933 del Parlamento Europeo y del Consejo, de 20 de mayo de 2019, por el que se modifica el Reglamento (CE) n. $.469 / 2009$ relativo al certificado complementario de protección para los medicamentos, introduce una excepción a la protección que confiere el certificado para permitir la fabricación de un producto, o un medicamento que contenga ese producto, para fines de exportación a terceros países o de almacenamiento. Dicha exención del CCP para fabricación se conoce en el sector como manufacturing SPC waiver.

En concreto, el Reglamento (UE) 2019/933 modifica el artículo 5 del Reglamento (CE) $n$.ㅇ 469/2009, de manera que se establece que el CCP no conferirá protección frente a determinados actos para los que de otro modo se requeriría el consentimiento del titular del certificado, que son:

- La fabricación de un producto, o un medicamento que contenga ese producto, para fines de exportación a terceros países, o cualquier acto conexo ${ }^{10}$ que sea estrictamente necesario para dicha fabricación en la Unión.

- La fabricación en los últimos 6 meses previos a la expiración del CCP, de un producto, o un medicamento que contenga ese producto, para su almacenamiento en el Estado miembro de fabricación, con el fin de comercializar dicho producto, o medicamento que contenga ese producto, en los Estados miembros después de la expiración del CCP, así como cualquier acto conexo a dicha fabricación. $D$

10 Por ejemplo, un acto conexo sería la importación de materiales de partida para el inicio de la fabricación.

El Reglamento (UE) 2019/933 promociona la competencia eliminando barreras legales para las empresas farmacéuticas, ubicadas en la UE, fabricantes de medicamentos genéricos y/o biosimilares en relación con:

- La exportación de medicamentos a mercados en países donde haya expirado la patente o no exista.

- La comercialización el día 1, tras expiración de CCP, en los mercados de la UE.

Fuente: elaboración propia. 


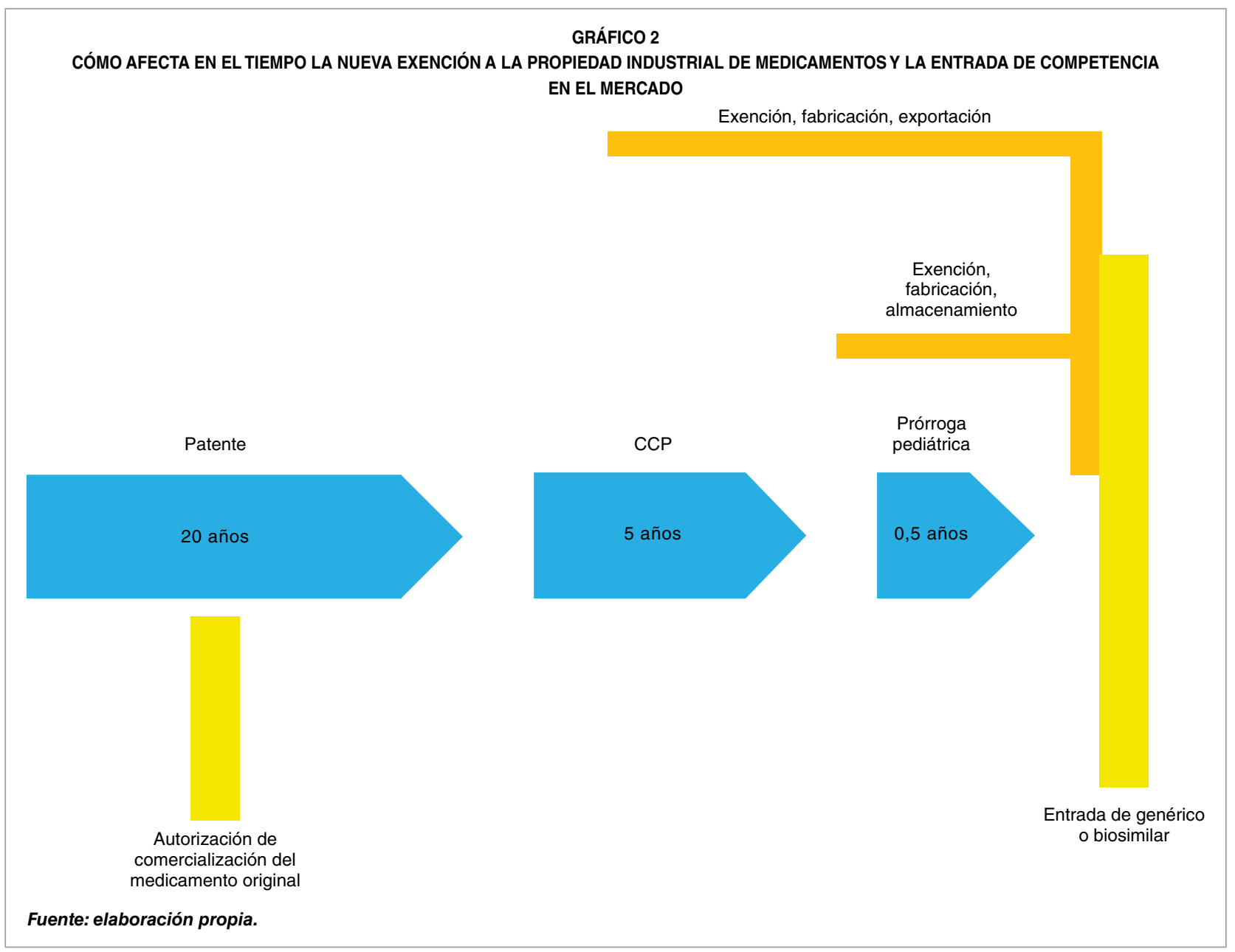

Como salvaguarda se introduce la obligación, para el fabricante, de comunicar el uso de dicha excepción al titular del certificado y a la autoridad competente en materia de propiedad industrial en el Estado miembro en el que se desarrollará la fabricación, que en España es la OEPM, con una antelación mínima de 3 meses del comienzo de la fabricación. También se señala la obligación del fabricante de garantizar que, quienes tienen relación contractual con él, estén plenamente informados del hecho de que la comercialización, la importación o la reimportación del producto podría suponer una infracción del certificado, al que se hace referencia en dicho apartado, en aquellos casos en los que se aplique el certificado y durante la validez del mismo.
Para dar cumplimiento a dicho deber de comunicación de dicha fabricación se prevé un formulario ${ }^{11}$ en el reglamento (Cuadro 2).

La contrapartida de fabricar, bajo la exención descrita, es que se comunica, por adelantado, a la empresa titular del CCP, que es un competidor, una buena parte del negocio a llevar y de la estrategia comercial de la empresa, como puede apreciarse en el Cuadro 2.

A esta salvaguarda, para las empresas innovadoras, se une que el medicamento fabricado para exportación dispondrá, en el embalaje exterior del producto, del logotipo $\triangle$

11 Formulario tipo de notificación de conformidad con el artículo 5, apartado 2, letras b) y c) del Reglamento (UE) 2019/933. 
CUADRO 2

FORMULARIO TIPO DE NOTIFICACIÓN

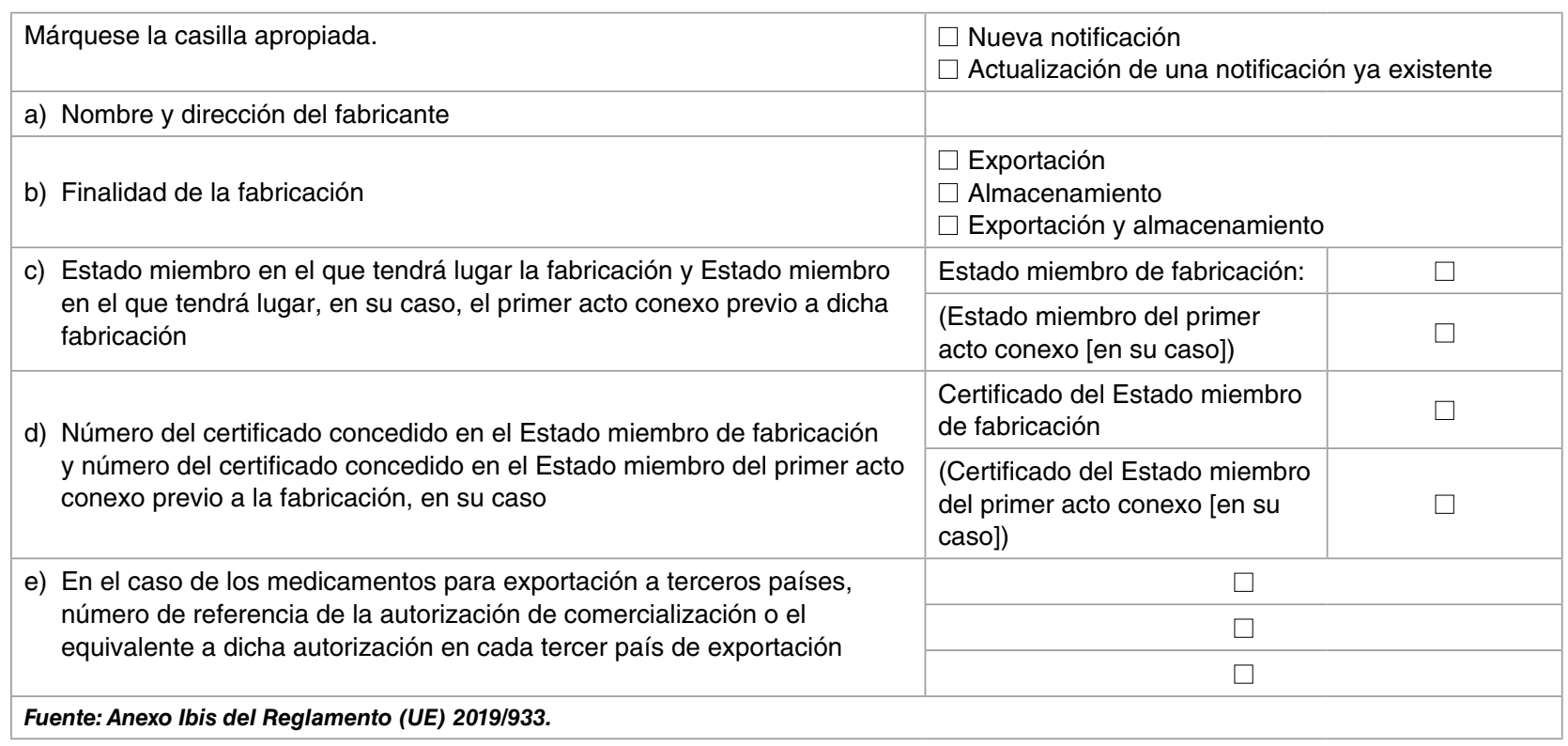

«EU export», que figura en el Anexo I del Reglamento. Así pues, el riesgo de desvío de los medicamentos fabricados para la exportación podrá controlarse con dicha medida de diferenciación de productos. A este respecto se puede tener en cuenta el precedente de fabricación de medicamentos esenciales destinados a los países en desarrollo (lucha contra el VIH/SIDA, la tuberculosis y la malaria) con riesgo de reimportación dado su precio diferenciado, drásticamente reducido. Entonces se diseñaron estrategias tales como apariencia distinta para facilitar su identificación ${ }^{12}$.

Por último, cabe destacar que la aplicación de este nuevo reglamento prevé un periodo transitorio para que los agentes en el mercado puedan adaptarse a la nueva normativa. Se señala en el preámbulo que para salvaguardar los derechos de los titulares de certificados, la excepción establecida en el presente reglamento no debe aplicarse a un certificado que

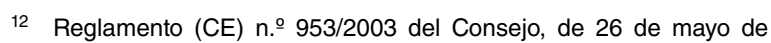
2003, destinado a evitar el desvío comercial hacia la Unión Europea de determinados medicamentos esenciales. ya haya surtido efecto en la fecha de entrada en vigor del reglamento.

La excepción debe aplicarse a los certificados solicitados en la fecha de entrada en vigor del reglamento, 1 de julio de 2019, o con posterioridad. Habida cuenta de que un certificado comienza a surtir efecto a la expiración del periodo de validez legal de la patente de base, lo que puede representar un periodo relativamente largo, y a fin de salvaguardar el objetivo del presente reglamento, está justificado que este también se aplique, durante un periodo determinado, a los certificados solicitados antes de la fecha de entrada en vigor del presente reglamento, pero que aún no han comenzado a surtir efecto antes de dicha fecha, y con independencia de si el certificado ha sido concedido o no antes de esa fecha. Por consiguiente, la excepción se aplica a partir del 2 de julio de 2022 a los certificados que comiencen a surtir efecto a partir del 1 de julio de 2019. Este periodo transitorio es fruto de una solución de consenso para equilibrar los intereses de los grupos de empresas afectados. 


\section{Efectos del cambio legislativo en España}

\subsection{La oferta en España de genéricos y biosimilares}

Por el lado de la oferta, el sector farmacéutico, tanto en España como en la UE, está compuesto por gran variedad de empresas que pueden clasificarse en tres bloques: grandes multinacionales, pymes especializadas en la venta de medicamentos no intensivos en I+D y biotecnológicas. En España, en concreto, conviven grandes multinacionales con grupos familiares y empresas spin-off de universidades o de parques tecnológicos. Según los datos publicados por el Instituto Nacional de Estadística (Encuesta Industrial de Empresas), el número de empresas del sector farmacéutico en España ascendió en 2017 a 353 y el número de personas ocupadas en la industria farmacéutica, a 42.653, y con una cifra de negocios de 13.541 millones de euros, similar a la de 2016.

El empleo en la industria farmacéutica con actividad en España se caracteriza por un perfil de elevada calidad y cualificación, con presencia mayoritaria de mujeres y una importante generación de empleo para jóvenes (Farmaindustria, 2018).

En cuanto a la localización de las empresas, se concentra en Madrid y Cataluña, sumando ambas el $79 \%$ de la cifra de negocios total del sector en nuestro país.

Las empresas farmacéuticas españolas, algunas muy significativas desde el punto de vista del desarrollo, no constituyen la mayoría, ni mucho menos. Las empresas farmacéuticas internacionales tienen una importante presencia, y muchas de ellas llevan a cabo actividades de investigación o de producción, o de ambas en nuestro país (Nombela, 2014).
España representa un importante mercado farmacéutico dentro de la UE, el quinto en volumen, como corresponde a la envergadura de nuestra población (Nombela, 2014).

Respecto a la producción, las empresas pueden dividirse en tres grupos según su actividad industrial: fabricante de materias primas, de medicamentos de uso humano o tanto de materias primas como de medicamentos de uso humano. De los resultados del programa Profarma se observa que el $57 \%$ de las empresas nacionales presentadas fabrican tanto materias primas como medicamentos de uso humano. Este porcentaje baja al $24 \%$ en el caso de compañías internacionales. Se observa también que el total de las empresas nacionales realizan actividades productivas en España, mientras que 14 empresas de capital internacional no disponen de planta de producción, si bien realizan actividades de I+D+i, dado que este es un requisito indispensable para su inclusión en el programa (Mincotur, 2018).

La industria farmacéutica española tuvo una tradición de fabricación de medicamentos «copia», porque hasta 1992 existía únicamente la patente de procedimiento (que no la patente de producto) (Segura, 1998; Laguna, 2006). Con el tiempo, esos medicamentos copia fueron pasando en su mayoría a autorizarse como medicamentos genéricos. Actualmente contamos con una industria de genéricos a nivel nacional bien establecida. De la industria farmacéutica de medicamentos genéricos en España, la mitad de las empresas cuentan con planta de producción propia y un tercio de las compañías fabrican además productos innovadores (AESEG, 2009). Un 70\% de los medicamentos genéricos consumidos en la actualidad en España se fabrican en nuestro país (AESEG, 2018). Esta industria se beneficiará de las nuevas oportunidades que presenta el nuevo $D$ 
reglamento, para el mercado de medicamentos genéricos, y podría en un futuro expandirse en el mercado de los biosimilares.

Si bien no debemos olvidar que existen importantes diferencias entre los productos genéricos y los biosimilares en cuanto a costes de desarrollo y de producción. En general, para el desarrollo de un medicamento genérico se requieren entre dos y tres años y una inversión de 1 a 3 millones de euros, mientras que para el desarrollo de un biosimilar se necesitan entre cinco y ocho años y de 100 a 300 millones de euros, según datos del «Libro Blanco de los Medicamentos Biosimilares en España: Innovación y Sostenibilidad» (Zaragozá et al., 2017).

Respecto a la fabricación de medicamentos biosimilares, existen actualmente en España dos laboratorios farmacéuticos fabricantes (García, 2016; Pmfarma, 2018). Adicionalmente, existen fabricantes de medicamentos en España que potencialmente podrían dedicarse a la fabricación de biosimilares. Un total de 34 laboratorios fabricantes o instalaciones de fabricación cuentan con la autorización de la AEMPS para la fabricación de medicamentos biológicos de uso humano en España, según datos de Labofar $^{13}$.

El desarrollo y/o la fabricación de un medicamento biosimilar parece estar más al alcance de las grandes empresas de medicamentos innovadores que de las pymes de medicamentos genéricos.

El mercado de aguas arriba, de fabricación de principios activos o ingredientes farmacéuticos activos en España, goza de una buena situación a nivel de comercio exterior. Así, el Reglamento (UE) 2019/933 abre nuevas oportunidades de negocio, que podrían beneficiar a

13 Labofar es el Registro de Laboratorios Farmacéuticos de la AEMPS. las empresas en España. La Comisión Europea señalaba en su análisis de impacto a las empresas fabricantes de principios activos para genéricos y biosimilares: «China es el líder mundial en fabricación y exportación de principios activos (APIs) por volumen, con un $20 \%$ del total del mercado de principios activos $y$ habiendo desplazado a India (fuente: http:// www. who.int/phi/publications/2081China 020517.pdf). En el pasado, se produjo una gran deslocalización de fabricación de principio activo a Asia, lo cual no creó una gran dependencia de Asia sobre principios activos para medicamentos que es una materia básica. De acuerdo con el informe CRA, Asia representa actualmente el $63 \%$ de la producción mundial de principios activos. La fabricación europea de principios activos se desarrolla principalmente en España e Italia, lo que representa el $21 \%$ del mercado mundial» (Comisión Europea, 2018).

La relevancia del sector español como proveedor de principio activo también aparecía en otro informe, encargado por la Comisión Europea. En concreto se señalaba que «entre los países fabricantes de principio activo en la UE, de los primeros genéricos en entrar en el mercado, Eslovenia era el proveedor más común (19\%), seguido de España (10\%) e Italia (7\%)» (CRA, 2016).

En relación con el comercio exterior, los productos farmacéuticos lideraron las exportaciones de productos químicos con un $33 \%$ durante el año 2016 (Mincotur, 2019). Los productos más importados son los medicamentos, con un $23,9 \%$ sobre el total de la cifra de negocios importada, con Alemania y EE UU como principales proveedores. Entre estos dos países acumulan el $40 \%$ de los medicamentos importados a nuestro país. En segundo lugar, se encuentran los hemoderivados de uso $D$ 
farmacéutico, que acumulan el 6,4\% de las importaciones, también con Alemania y EE UU a la cabeza de las exportaciones de estos productos a España.

Por último, señalar que, para el crecimiento de la industria farmacéutica, España dispone de una estructura de apoyo a la inversión y el desarrollo, ya sea para nuevas empresas o para empresas existentes que quieren acometer mejoras. El organismo Invest in Spain, dependiente de la entidad pública ICEX España Exportación e Inversiones, cuenta con un servicio de Atracción de Inversión a España en el sector de la biotecnología, farmacia y ciencias de la vida (ICEX, 2019).

\subsection{La demanda en España de genéricos y biosimilares}

Por el lado de la demanda, España representa un importante mercado farmacéutico dentro de la UE en volumen de compra.

El Servicio Nacional de Salud (SNS) se beneficiará de la pronta entrada de medicamentos genéricos y biosimilares de empresas establecidas en la UE, con un aumento de la seguridad y de la calidad en el suministro, como, por ejemplo, una menor incertidumbre debido a la dependencia de las importaciones. La Comisión Europea ha puesto de relieve la importancia de la seguridad en el suministro para las oficinas de farmacia, entre otros, en el análisis de la operación de concentración Teva/ Allegan Generics, donde llegó a imponerse como compromiso la desinversión de una fábrica en la UE para mantener la competencia efectiva en el mercado.

En los mercados que comercializan medicamentos genéricos, el ahorro medio para el sistema sanitario (considerando un índice ponderado de precios de productos originales y genéricos) alcanza casi el $20 \%$ en el primer año transcurrido tras la llegada al mercado del genérico y aproximadamente el $25 \%$ en los dos primeros años, media de la UE (Comisión Europea, 2009).

Se estima que el ahorro generado en el SNS español por la introducción de los biosimilares en el periodo 2009-2016 ascendió a 478 millones de euros. La mayor parte del ahorro total producido en ese periodo se debió a la introducción del biosimilar de la insulina glargina (25\%), seguido del de somatropina (21\%), infliximab (20\%), filgrastim (16\%) y epoetina (13\%) (Zaragozá et al., 2017) ${ }^{14}$. Se proyecta aún un ahorro mayor. Según estudio de la Fundación Weber, se estima que los ahorros derivados de los biosimilares entre 2017 y 2020 cuadruplicarían a los alcanzados durante los ocho años anteriores, gracias a la mayor penetración de los biosimilares, pero sobre todo debido a la expiración de las patentes en grandes nichos de mercado. Así, se estima que el uso de los biosimilares generaría unos ahorros totales superiores a los 2.400 millones de euros entre 2009 y 2020 en España (González, 2017).

En el conjunto mundial, a lo largo de la última década se han comercializado unas 80 moléculas biológicas, y se estima que hasta 2020 se comercializarán otras 60-70. Esto es una muestra del extenso futuro mercado potencial que se abrirá a los biosimilares con la caducidad de las patentes de sus biológicos de referencia. Sólo en España, el valor acumulado de mercado de los ocho biosimilares que perderán su exclusividad entre 2016 y 2020 es de unos 5.000 millones de euros. A lo que hay que añadir que el número de biosimilares en $\triangleright$

14 Véase en particular el capítulo «El mercado de los medicamentos biosimilares en España: acceso y sostenibilidad. ¿Qué ha supuesto la introducción de los biosimilares en España 10 años después?». 
fase de desarrollo también es un indicativo del auge previsto en este mercado (Zaragozá et al., 2017).

El principal valor añadido atribuible a la entrada de los biosimilares es la posibilidad de que un mayor número de pacientes acceda a medicamentos biológicos ${ }^{15}$. La reducción del coste, respecto al producto original, permite la incorporación de los medicamentos biosimilares a los sistemas sanitarios.

La competencia es el principal motor del ahorro final en costes producidos por la entrada de los biosimilares al mercado, así como para la investigación y desarrollo de nuevos biosimilares.

Las autoridades de competencia europea y nacionales velan por la competencia en este sector, siendo de interés la intervención de la Comisión Europea en la operación de concentración Pfizer/Hospira, dado que ambas empresas tenían biosimilares en fase III de desarrollo. En particular, se observaron problemas de competencia en el mercado de biosimilares de infliximab, por los que Pfizer se comprometió a la desinversión de su infliximab en desarrollo, que incluía su desarrollo, fabricación y derechos de comercialización, para asegurar la competencia efectiva en dicho mercado en la UE (Mazurkiewicz, 2016).

\section{Conclusiones}

Se puede pensar que los beneficios que trae el nuevo reglamento para los fabricantes europeos y los sistemas sanitarios son más relevantes que los posibles perjuicios para los laboratorios innovadores. Las exenciones al CCP

15 Los medicamentos biológicos son un alternativa terapéutica a los habituales medicamentos de síntesis química, pero de una mayor complejidad en su producción y de un mayor precio. que establece el reglamento no son contrarias a la exclusividad en el mercado de la UE que se le ha otorgado a la empresa para un medicamento. Las empresas serán conocedoras del uso de la exención a través del sistema de notificación, así como las autoridades de patentes, y los productos irán identificados de manera que se impida el desvío.

La nueva regulación podría parecer que establece beneficios en el mercado para un determinado tipo de empresas farmacéuticas, aquellas fabricantes de genéricos y/o biosimilares frente a las productoras de medicamentos innovadores. En la práctica no es probable tal enfrentamiento, ya que el desarrollo de productos biosimilares requiere una infraestructura y una investigación previa que solo las grandes empresas tienen ya establecidas, creándose de este modo nuevas expectativas en cuanto a la mejora industrial de todas las empresas farmacéuticas ubicadas en la UE.

\section{Agradecimientos}

A Luis González Calbet y Blanca Fernández Barjau, Ministerio de Economía y Empresa.

\section{Bibliografía}

AESEG (2018). 20 años del genérico en España, 1997-2017. En Genérico (23).

Bailly, M., Gasperec, I., Stril, A., y Laguna-Goya, N. (2016). Teva/Allergan Generics. Competition Merger Brief (2). Recuperado de https://ec.europa.eu/competition/publications/cmb/2016/ cmb2016_002_en.pdf

Charles Rivers Associates (2016). Informe CRA: Assessing the economic impacts of changing exemption provisions during patent and SPC $\square$ 
protection in Europe. Recuperado de https://publications.europa.eu/en/publication-detail/-/ publication/6e4ce9f8-aa41-11e7-837e-01aa75ed71a1/language-en

Comisión Europea (2009). Informe final de «Pharmaceutical Sector Inquiry» de 8 de julio. Recuperado de https://ec.europa.eu/competition/sectors/pharmaceuticals/inquiry/

Comisión Europea (2015). COM(2015) 550, Comunicación de la Comisión al Parlamento europeo, al Consejo, al Comité Económico y Social europeo y al Comité de las Regiones: "Mejorar el mercado único: más oportunidades para los ciudadanos y las empresas». Recuperado de https://eur-lex.europa.eu/legal-content/ES/TXT/ PDF/?uri=CELEX:52015DC0550\&from=EN

Comisión Europea (2018). Impact Assessment accompanying the Proposal for a Regulation COM(2018) 317. SWD(2018) 240. Recuperado de https://ec.europa.eu/docsroom/documents/ 29463

Comisión Europea (2019). Informe de la Comisión al Consejo y al Parlamento europeo. Aplicación de las normas de competencia en el sector farmacéutico (2009-2017). Colaboración entre las autoridades europeas de competencia en favor de unos medicamentos asequibles e innovadores. Recuperado de http://ec.europa.eu/competition/sectors/pharmaceuticals/report2019/ report_es.pdf

Comisión Europea (17 de abril, 2019). Targeted adjustment of intellectual property rules for pharmaceuticals. (Nota de prensa). Recuperado de https://europa.eu/rapid/press-release_ MEMO-19-2179_en.htm

Consejo de la UE (16 de enero, 2019). La UE contribuirá a impulsar las exportaciones de medicamentos genéricos. (Comunicado de prensa). Recuperado de https://www.consilium.europa.eu/ es/press/press-releases/2019/01/16/eu-to-helpboost-exports-of-generic-pharmaceuticals/

Consejo de la UE (20 de enero, 2019). Medidas de la UE en favor de los productores de medicamentos genéricos. (Comunicado de prensa). Recuperado de https://www.consilium.europa.eu/es/press/ press-releases/2019/02/20/eu-measures-in-supportof-generic-pharmaceuticals-producers/

Consejo de la UE (14 de mayo, 2019). Medidas de la UE en favor de los productores de medicamentos genéricos. (Comunicado de prensa). Recuperado de https://www.consilium.europa. eu/es/press/press-releases/2019/05/14/eu-adoptsmeasures-in-support-of-generic-pharmaceuticalsproducers/

Farmaindustria (4 de octubre, 2018). La industria farmacéutica innovadora supera ya los 40.500 empleos directos, el $94 \%$ indefinidos. (Nota de prensa). Recuperado de http://www.farmaindustria.es/web/prensa/notas-de-prensa/2018/10/04/laindustria-farmaceutica-innovadora-supera-yalos-40-500-empleos-directos-el-94-indefinidos/

García Arieta, A., Hernández García, C., y Avendaño Solá, C. (2010). Regulación de los medicamentos genéricos: evidencias y mitos. Información Terapéutica del Sistema Nacional de Salud 34 (3).

Garcia, M. (4 de septiembre, 2016). La planta de mAbxience en León culmina la fabricación de su primer lote de biosimilares. El Global. Recuperado dehttps://www.elglobal.es/hemeroteca/la-plantade-mabxience-en-leon-culmina-la-fabricacion-desu-primer-lote-de-biosimilares-BAEG_988911

González, A., Ivanona, Y., Zozaya, N., Jiménez, M., e Hidalgo, A. (2017). La introducción de los biosimilares en España. Estimación del ahorro para el Sistema Nacional de Salud. Madrid, España: Fundación Weber.

ICEX (2019). Ficha de Ayudas e incentivos: Sector Biotecnología, Farmacia y Ciencias de la vida. Recuperado de http://www.investinspain.org/ invest/es/canal-de-informacion/documentacion/como-hacer-negocios-en-espana/ DOC2018797823.html

Laguna Goya, N. (2012). El sector farmacéutico desde el punto de vista de la competencia. Comun. Prop. Ind. Derecho Competencia (65), 213-253. 
Laguna Goya, N., Blázquez Pérez, A., y Pozo Hernández, C. (2006). Legislación sobre autorización de genéricos. Farmacia Hospitalaria 30(6), 379-384.

Mazurkiewicz, A., y Stril, A. (2016). Pfizer / Hospira: Through the looking-glass: assessing competition by biosimilars. Competition Merger Brief (1). Recuperado de http://ec.europa.eu/competition/ publications/cmb/2016/cmb2016_001_en.pdf

Mincotur (2018). Informe de resultados de la convocatoria 2017 de PROFARMA (2017-2020): fomento de la competitividad en la industria farmacéutica.

Mincotur (2019). Agenda Sectorial de la Industria Química y del Refino en España.

Navarro Espigares, J. L., y Hernández Torres, E. (2007). Industria farmacéutica, competitividad e integración económica en Europa. Boletín Económico de ICE, (2902).

Nombela C. (2014). Investigación e industria farmacéutica. Pilar de salud. Cincuenta años de Economía Industrial. Economía Industrial (394). Recuperado de https://www.mincotur.gob.es/Publicaciones/Publicacionesperiodicas/Economia Industrial/RevistaEconomialndustrial/394/ CESAR\%20NOMBELA.pdf

OCDE (2009). Policy roundtable: Generic Pharmaceuticals. DAF/COMP(2009)39. Recuperado de http://www.oecd.org/competition/sectors/46138891.pdf

Parlamento Europeo (2019). European Parliament legislative resolution of 17 April 2019 on the proposal for a regulation of the European Parliament and of the Council amending Regulation (EC)
No. 469/2009 concerning the supplementary protection certificate for medicinal products (COM(2018)0317 - C8-0217/2018 - 2018/0161 (COD)). Recuperado de http://www.europarl.europa.eu/doceo/document/TA-8-2019-0401_EN. html? redirect\#top

Pmfarma (2018). Rovi ha iniciado la comercialización de su biosimilar de enoxaparina en España. Recuperado de http://www.pmfarma.es/noticias/ 26206-rovi-ha-iniciado-la-comercializacion-desu-biosimilar-de-enoxaparina-en-espana.html

Reglamento (UE) 2019/933 del Parlamento Europeo y del Consejo, de 20 de mayo de 2019, por el que se modifica el Reglamento (CE) n. $-969 / 2009$ relativo al certificado complementario de protección para los medicamentos. Diario Oficial de la Unión Europea L 153/1-10, de 11 de junio de 2019. Recuperado de https:// www.boe.es/doue/2019/153/L00001-00010.pdf

Sauri, LI. (2015). Desarrollos recientes en la política de la competencia en el sector farmacéutico: Más competencia para una mayor inversión en innovación y para un mejor acceso a los fármacos. Papeles de Economía Española (145), 197-211.

Segura, P. (1998). Patentes y medicamentos genéricos en España. Revista Española de Farmacoeconomía, diciembre, 13-19.

Rodrigo, J. (2019). Medicamentos, innovación tecnológica y economía. Papeles de Economía Española (160), 196-203.

Zaragozá, F., Villaescusa L., y Pi Corrales, G. (2017). Libro Blanco de los Medicamentos Biosimilares en España. Madrid, España: Fundación Gaspar Casal (2. ${ }^{a}$ edición). 